\title{
Prospective follow-up study of children with univentricular heart: Neurodevelopmental outcome at age 12 months
}

Anne Sarajuuri, MD, ${ }^{a}$ Tuula Lönnqvist, $\mathrm{MD}, \mathrm{PhD},{ }^{\mathrm{a}}$ Leena Mildh, MD, PhD, ${ }^{\mathrm{b}}$ Irmeli Rajantie, $\mathrm{PT},{ }^{\mathrm{a}}$ Marianne Eronen, $\mathrm{MD}, \mathrm{PhD},{ }^{\mathrm{c}}$ Ilkka Mattila, $\mathrm{MD}, \mathrm{PhD},{ }^{\mathrm{d}}$ and Eero Jokinen, $\mathrm{MD}, \mathrm{PhD}^{\mathrm{c}}$

Objectives: Despite recent advances in the treatment of children with univentricular heart, their neurodevelopmental outcome remains a major concern.

Methods: This prospective follow-up study evaluated the neurodevelopmental outcome of 23 patients with hypoplastic left heart syndrome, 14 with other forms of univentricular heart, and 46 healthy control subjects at a median age of 12.2 months. The Griffiths Developmental Scale and Alberta Infant Motor Scale served for developmental evaluation.

Results: The mean Griffiths developmental quotient of children with hypoplastic left heart syndrome was significantly less $(91.6)$ than that of control children $(106.8, P<.001)$. Patients with univentricular heart scored significantly lower than control subjects only in the gross motor domain $(P=.001)$ but not in overall development (100.6). Alberta Infant Motor Scale scores were significantly lower in children with hypoplastic left heart syndrome $(37.5, P<.001)$ and univentricular heart (43.5, $P=.011)$ than in control subjects (53.3). In linear regression a diagnosis of hypoplastic left heart syndrome $(P=.016)$, a clinical history of seizure $(P=.002)$, and the highest plasma lactate level after the bidirectional Glenn operation $(P=.045)$ were significantly associated with the developmental quotient.

Conclusions: At age 1 year, the level of development of children with univentricular heart was significantly lower than for control subjects only in motor skills, whereas children with hypoplastic left heart syndrome had a more widespread developmental delay. The diagnosis, a clinical seizure history, and increased plasma lactate levels after the bidirectional Glenn operation emerged as risk factors.

Supplemental material is available online.

With progress in surgical and perioperative care, the survival rate of children with hypoplastic left heart syndrome (HLHS) and other forms of univentricular heart (UVH) has increased. ${ }^{1}$ Consequently, the survivors' neurodevelopment has become a focus of interest. Recent surgical advances include right ventricle-pulmonary artery (RV-PA) shunt in the Norwood operation ${ }^{2}$ and the implementation of regional low-flow perfusion (RLFP) to replace deep hypothermic cardiac arrest (DHCA). ${ }^{3}$ The risk for cerebral damage increases significantly if the duration of DHCA exceeds 40 to 45 minutes, ${ }^{4}$ which was often the case in the Norwood operation earlier. The Boston Circulatory Arrest

\footnotetext{
From the Divisions of Neurology ${ }^{a}$ Anesthesiology and Intensive Care Medicine ${ }^{\mathrm{b}}$ Paediatric Cardiology, ${ }^{\mathrm{c}}$ and Surgery ${ }^{\mathrm{d}}$ Department of Paediatric and Adolescent Medicine, Helsinki University Central Hospital, Helsinki, Finland.

Supported by grants from the Arvo and Lea Ylppö Foundation, the Lastenlinna Foundation, the Foundation for Pediatric Research, and the Finnish Foundation for Cardiovascular Research.

Received for publication Jan 6, 2008; revisions received April 1, 2008; accepted for publication June 15, 2008.

Address for reprints: Anne Sarajuuri, MD, Department of Child Neurology, Department of Paediatric and Adolescent Medicine, Helsinki University Central Hospital,

Lastenlinnantie 2, 00029 HUS, Finland (E-mail: Anne.Sarajuuri@hus.fi).

J Thorac Cardiovasc Surg 2009;137:139-45

$0022-5223 / \$ 36.00$

Copyright (c) 2009 by The American Association for Thoracic Surgery

doi:10.1016/j.jtcvs.2008.06.025
}

Study, evaluating children with transposition of the great arteries, showed that compared with the use of RLFP, DHCA was associated with worse neurologic outcome, especially in the motor area. ${ }^{4}$ On the other hand, such an association has not been found in 2 recent studies comparing DHCA and RLFP among patients who underwent the Norwood operation. ${ }^{5,6}$ Use of an RV-PA shunt has been associated with faster recovery after the Norwood operation, ${ }^{7}$ although no significant difference in mortality has been found. ${ }^{8}$ Studies comparing neurodevelopmental outcome are lacking. In addition to these intraoperative factors, several preoperative and postoperative factors increase the neurologic risks to patients: structural brain anomalies, low preoperative cerebral blood flow, postoperative hemodynamic instability, chronic hypoxemia, and cerebrovascular accidents.

In most studies of children with HLHS operated on in the 1990's, mean intelligence has remained in the low average range. ${ }^{9-12}$ Only a minority of patients has more serious neurologic sequelae, such as cerebral palsy or mental retardation, but specific learning difficulties are common. To assess more carefully the neuropsychologic profile to determine the risk for learning difficulties requires comparison with healthy control children, which has not been done in most previous studies.

\section{MATERIALS AND METHODS \\ Patient Population}

Children with HLHS or other forms of UVH born between August 2002 and February 2005 and operated on at the Department of Paediatric and Adolescent Medicine of the Helsinki University Central Hospital were offered 


$$
\begin{aligned}
& \text { Abbreviations and Acronyms } \\
& \begin{aligned}
\text { AIMS } & =\text { Alberta Infant Motor Scale } \\
\text { BDG } & =\text { bidirectional Glenn operation } \\
\text { BT } & =\text { Blalock-Taussig } \\
\text { DHCA } & =\text { deep hypothermic cardiac arrest } \\
\text { DQ } & =\text { developmental quotient } \\
\text { EEG } & =\text { electroencephalography } \\
\text { HLHS } & =\text { hypoplastic left heart syndrome } \\
\text { RLFP } & =\text { regional low-flow perfusion } \\
\text { RV-PA } & =\text { right ventricle-pulmonary artery } \\
\text { SD } & =\text { standard deviation } \\
\text { UVH } & =\text { univentricular heart }
\end{aligned}
\end{aligned}
$$

the possibility to participate in a prospective neurodevelopmental follow-up study. All pediatric cardiac surgery in the Finnish population of 5.3 million is performed at this hospital. During this time period, 2 patients with HLHS were not actively treated. The number of eligible children with HLHS/UVH, after the exclusion of 1 patient because of Cantrell's pentalogy and 2 because of a chromosomal defect, was 52. Nine patients were not recruited because of a lack of parental consent. This meant 28 children with HLHS and 15 children with other forms of UVH were recruited. Control children numbered 46 , with a similar age and sex distribution $(64.9 \%$ boys among the patients and $65.2 \%$ among the control subjects). The control children were recruited from low-risk deliveries at the Department of Obstetrics and Gynecology of Helsinki University Central Hospital after a pediatric check-up with normal findings. Their normal cardiac anatomy was confirmed with ultrasonographic analysis. The study was approved by the Ethics Committee of the Department of Paediatric and Adolescent Medicine at the Helsinki University Central Hospital. Written informed consent of all parents was obtained.

\section{Neurodevelopmental Assessment}

A routine neurologic examination of all subjects was performed at a median age of 12.2 months, with assessment of muscle tone (level and symmetry), deep tendon reflexes, functions of the cranial nerves (pupillary reactions, possible strabismus, eye movements, symmetry of mimics and pharynx, localization of sound, and head turning), side and back protection reflexes from a sitting position, and parachute reaction. The findings were classified as either normal or abnormal, except for muscle tone, which was graded as either normal, mild hypotonia, severe hypotonia, or hypertonia. The neurologic findings were classified as mildly abnormal if aberrant findings existed in 1 or 2 compartments and clearly abnormal for aberrant findings in more than 2 compartments. As a common finding, mild muscle hypotonia was not included in this grading of neurologic abnormality. Head circumference, weight, and length were recorded and compared with Finnish national standards. ${ }^{13,14}$ Microcephaly was diagnosed if head circumference was more than 2 standard deviations (SDs) below the mean for the age- and sex-specific population standard. The Griffiths developmental scale ${ }^{15}$ served for developmental assessment, and the Alberta Infant Motor Scale (AIMS) ${ }^{16}$ served for a more detailed motor evaluation. A Griffiths developmental quotient (DQ) was determined according to the test manual if all 5 subdomains (gross motor, social, verbal, and visuomotor development and performance) could be adequately evaluated. The motor assessments were recorded on videotape and were analyzed according to the AIMS manual by 2 physiotherapists well experienced in child developmental assessment.

\section{Surgical and Perfusion Strategies}

The aortic reconstructions during Norwood-type operations and possible additional aortic reconstructions caused by aortic recoarctations were performed during either RLFP alone or in combination with short periods of DHCA (maximum of 9 minutes), and 1 patient underwent a correction of the superior vena cava with a DHCA of 20 minutes. During surgical corrections, the perfusion strategies were as follows. With the Norwood I procedure and all other operations involving aortic arch reconstruction, (1) the hematocrit value was kept at $0.30 \%$, (2) cooling was aimed at $18^{\circ} \mathrm{C}$, and (3) $\alpha$-stat $\mathrm{pH}$ management was used, and during RLFP, the pump flow was kept between 15 and $20 \mathrm{~mL} \cdot \mathrm{kg}^{-1} \cdot \mathrm{min}^{-1}$. With the bidirectional Glenn operation (BDG), hematocrit values were also aimed at $0.30 \%$, but the body temperature was kept between $30^{\circ} \mathrm{C}$ and $32^{\circ} \mathrm{C}$. Toward the end of perfusion, ultrafiltration was performed, if needed, to increase the hematocrit value. During BDG, the superior vena cava was cannulated distally, almost at the junction of the jugular and anonymous veins. Another cannula was placed to the right atrial appendix except when intracardial procedures were performed; then it was placed to the inferior vena cava.

\section{Clinical Data Collection}

The medical records were analyzed concerning the operations performed and the durations of cardiopulmonary bypass, DHCA, RLFP, aortic crossclamping, cooling, and rewarming and the level and duration of hypothermia. The levels of diuresis (in milliliters per kilogram per hour) during the first 3 postoperative days and the highest plasma concentration of lactate (in millimoles per liter) during the postoperative time intervals of 0 to 6 , 6 to 24,24 to 48 , and 48 to 72 hours were recorded. Possible clinical seizures and resuscitations were documented. Electroencephalography (EEG) was performed on clinical indication for those with suspected convulsions. Of the EEG findings, focal abnormality was considered significant (either focal spikes/discharges or asymmetry). Perinatal data concerning Apgar scores at 1, 5, and 10 minutes and weight, height, and head circumference at birth were collected. Maternal and paternal occupation, ${ }^{17}$ as well as level of education, ${ }^{18}$ were recorded and classified according to the Finnish versions of these classifications. All these data were analyzed for association with the primary outcome measure, Griffiths DQ, in the univariate analysis.

\section{Statistical Analysis}

SPSS version 13.0 software (SPSS, Inc, Chicago, Ill) was used for statistical analysis. The Mann-Whitney test served for comparison of continuous variables between patients and control subjects. Kruskal-Wallis and Dunn tests were used for continuous data, and the Fisher's exact test was used for binary data to compare the results between the subgroups (children with HLHS, children with UVH, and control subjects). The Bonferroni correction was made for Fisher's $P$ values to correct for multiple comparisons. Pearson correlation coefficients were computed as a measurement of association with DQ for normally distributed continuous factors, and Spearman correlation coefficients were computed for categorical and nonnormally distributed continuous factors. Natural logarithm and exponential transformations were performed for those nonnormally distributed continuous factors that approached significance. All factors significantly associated with DQ were added into the linear regression model.

\section{RESULTS \\ Patient Characteristics}

Five of the recruited patients (4 with HLHS and 1 with $\mathrm{UVH}$ ) died before the age of 12 months. One patient was unable to participate in the follow-up visit at 12 months because of a tracheostomy caused by bilateral recurrent nerve paralysis and inability to travel long distances. At a median age of 12.2 months, 37 surviving patients and 46 healthy control children were examined. The diagnoses 
and operations performed are presented in Table E1. During our study, both the modified Blalock-Taussig (BT) shunt (13 patients) and the RV-PA shunt (15 patients) were used. Before the study visit, all children but 1 had undergone the BDG operation at a median age of 5.7 months, 8 patients needed a reconstruction of the aortic arch in connection with the BDG operation, and 6 children had experienced 1 and 2 patients had experienced 2 additional operations. The median support times are presented in Table 1. Extracardiac malformations were found in 3 study patients: diaphragmal hernia and asplenia related to heterotaxy, scoliosis, and vertebral anomalies, as well as a sensorineural hearing deficit.

\section{Developmental Assessment}

The mean Griffiths DQ could be adequately evaluated for 32 patients and 46 control children. The DQ was 95.0 in all patients with congenital heart defects compared with 106.8 in control subjects $(P<.001)$. The difference from control subjects was significant for children with HLHS but not for children with UVH (Table 2). Of the developmental domains, those with UVH were scored at a significantly lower level than control subjects only in the gross motor domain (29.5 vs $34.7, P=.004$ ), whereas the children with HLHS were scored at a significantly lower level than control children in the gross motor ( 27.0 vs $34.7, P<.001$ ), social (30.1 vs $33.7, P<.001)$, visuomotor ( 28.5 vs $32.0, P=.015)$, and performance domains (29.2 vs $32.9, P<.001$; Figure 1$)$.

The mean results in the AIMS motor developmental assessment were significantly better for the control subjects (53.3 points; SD, 4.5) than for either children with HLHS (37.5 points; SD, $14.5 ; P<.001$ ) or children with $\mathrm{UVH}$
(43.5 points; SD, $13.1 ; P=.011$; Figure 2 ). The AIMS results correlated significantly with Griffiths DQ results $(P<.001)$ and with Griffiths gross motor $(P<.001)$, social $(P=.005)$, and performance $(P=.011)$ domains.

\section{Neurologic Outcome}

Muscle hypotonia was a common finding among the children with HLHS/UVH $(70.3 \%$ compared with $19.6 \%$ in healthy control subjects, $P<.001$ ). It was mild in all control children, whereas 4 patients had severe muscle hypotonia, and 1 had muscle hypertonia. Asymmetric muscle tone was found in $17.4 \%$ of the children with HLHS (widespread in 1 and mild and local in 3) but in none of the control subjects or the children with UVH. The results of the neurologic examination when muscle hypotonia was excluded were abnormal in 10 patients with HLHS (43.5\%), 3 patients with UVH (21.4\%, not significant), and 1 control child (2.2\%, $P<.001$ for the difference from HLHS). The abnormality was mild in all but 3 patients with HLHS (13.0\%). Cerebral palsy resulting from a perioperative cerebral infarct was found: the form of hemiplegia in $1(4.3 \%)$ of the children with HLHS, and in the form of a hemiparesis in $1(7.1 \%)$ of the children with UVH. Ten $(27.0 \%)$ patients had received physiotherapy and 1 received speech therapy because of eating problems before the age of 12 months; 1 control child received physiotherapy because of a mildly delayed motor development.

\section{Growth Data}

The means of birth weight (3520 vs $3510 \mathrm{~g})$, height (50.4 $\mathrm{cm}$ in both groups), and head circumference (35.4 vs 35.3

TABLE 1. Median support times in the operations

\begin{tabular}{|c|c|c|c|c|c|c|c|c|c|c|c|c|c|c|c|}
\hline & \multicolumn{3}{|c|}{ CPB (min) } & \multicolumn{3}{|c|}{ RLFP time (min) } & \multicolumn{3}{|c|}{ DHCA time (min) } & \multicolumn{3}{|c|}{ Duration of cooling (min) } & \multicolumn{3}{|c|}{ Duration of rewarming (min) } \\
\hline & Median & Range & $\mathbf{n}$ & Median & Range & $\mathbf{n}$ & Median & Range & $\mathbf{n}$ & Median & Range & $\mathbf{n}$ & Median & Range & $\mathbf{n}$ \\
\hline \multicolumn{16}{|c|}{ Primary operation } \\
\hline HLHS & 190.0 & $137-252$ & 23 & 71.5 & $40-106$ & $22 *$ & 5.0 & $2-9$ & 10 & 30.0 & $10-60$ & 23 & 35.0 & $20-95$ & 23 \\
\hline UVH & 175.0 & $116-181$ & 5 & 61.0 & $42-81$ & 5 & 8.0 & 8 & 1 & 20.0 & $15-35$ & 5 & 35.0 & $30-50$ & 5 \\
\hline \multicolumn{16}{|c|}{ BDG operation } \\
\hline HLHS & 88.0 & $48-232$ & 23 & 22.0 & $8-50$ & 6 & & & & 30.0 & $5-85$ & 9 & 50.0 & $30-80$ & 9 \\
\hline UVH & 85.0 & $65-176$ & 12 & 17.0 & $16-32$ & 3 & & & & 25.0 & $15-50$ & 5 & 30.0 & $25-40$ & 5 \\
\hline \multicolumn{16}{|c|}{$\begin{array}{l}\text { Additional } \\
\text { operation }\end{array}$} \\
\hline HLHS & 97.5 & 34-132 & 4 & & & & & & & & & & & & \\
\hline UVH & 93.0 & $81-139$ & 3 & & & & & & & & & & & & \\
\hline \multicolumn{16}{|c|}{$\begin{array}{c}\text { Second additional } \\
\text { operation }\end{array}$} \\
\hline HLHS & 120.0 & $92-148$ & 2 & 29.0 & 29 & 1 & 20.0 & 20 & 1 & & & & & & \\
\hline \multicolumn{16}{|c|}{ Cumulative times } \\
\hline HLHS & 277.0 & $221-485$ & 23 & 72.0 & $18-151$ & 23 & 5.0 & $2-20$ & 11 & & & & & & \\
\hline UVH & 211.0 & $65-351$ & 12 & 77.0 & $55-102$ & 5 & 8.0 & 8 & 1 & & & & & & \\
\hline
\end{tabular}

$C P B$, Cardiopulmonary bypass; $R L F P$, regional low-flow perfusion; $D H C A$, deep hypothermic cardiac arrest; $H L H S$, hypoplastic left heart syndrome; $U V H$, univentricular heart; $B D G$, bidirectional Glenn operation. * One patient with HLHS was managed during the Norwood operation with a perfusion in which the right upper body, carotid artery, base of the ascending aorta, and lower body through the aorta were perfused. 
TABLE 2. Griffiths developmental quotient results in children with hypoplastic left heart syndrome, children with other forms of univentricular heart, and control subjects

\begin{tabular}{|c|c|c|c|}
\hline & HLHS $(n=20)$ & UVH $(\mathbf{n}=12)$ & Control subjects $(n=46)$ \\
\hline Mean Griffiths developmental quotient & 91.6 & 100.6 & 106.8 \\
\hline$P$ value for difference from control subjects & $<.001$ & NS & \\
\hline$P$ value for difference between children with HLHS and children with UVH & NS & & \\
\hline $\begin{array}{l}\text { Proportion of children with a developmental quotient }>1 \text { SD lower than the } \\
\text { mean of the control subjects }\end{array}$ & $15 / 20(75.0 \%)$ & $4 / 12(33.3 \%)$ & $10 / 46(21.7 \%)$ \\
\hline$P$ value for difference from control subjects & .002 & NS & \\
\hline$P$ value for difference between children with HLHS and children with UVH & NS & & \\
\hline $\begin{array}{l}\text { Proportion of children with a developmental quotient }>2 \text { SDs lower than the } \\
\text { mean of the control subjects }\end{array}$ & $7 / 20(35.0 \%)$ & $2 / 12(16.7 \%)$ & $0 / 46(0 \%)$ \\
\hline$P$ value for difference from control subjects & .003 & NS & \\
\hline$P$ value for difference between children with HLHS and children with UVH & NS & & \\
\hline
\end{tabular}

$\mathrm{cm} ; 35.5 \mathrm{~cm}$ in children with HLHS and $34.8 \mathrm{~cm}$ in children with UVH) did not differ significantly between patients and control subjects. One $(7.1 \%)$ patient with UVH and 2 $(8.7 \%)$ patients with HLHS had congenital microcephaly. Those 2 patients with HLHS and microcephaly at birth had a head circumference within normal limits at 12 months of age, whereas 2 other patients with HLHS had become and 1 patient with UVH remained microcephalic. As a group, the patients with HLHS/UVH had a significantly smaller mean head circumference than did the control children at the study visit (age- and sex-adjusted head circumference, -0.776 vs -0.111 [SD], $P=.001$ ), with no significant difference between those with HLHS and those with UVH. By contrast, mean height and weight did not differ significantly between the patients and control subjects.

\section{Occurrence of Seizures}

Eight $(21.1 \%)$ of the surviving patients (5 with HLHS and 3 with UVH) had clinical seizures with focal EEG abnormality during follow-up. Six patients had seizures during the postoperative period after the Norwood operation, 3 of them also with neuroradiologic evidence of cerebral injury. In 2 patients brain magnetic resonance imaging showed wide cerebral atrophy at the age of 2 months. They both presented with global developmental delay and abnormal eye movements with strabismus in the study assessment. The third child had ischemic changes in the watershed area on magnetic resonance imaging at age 17 months; she also has a bilateral sensorineural hearing deficit and vertebral anomalies. The 3 other patients with seizures during their recovery from the Norwood operation showed no other evidence of neurologic injury. In addition, after the Norwood operation, 1 patient with HLHS had a suspected mild facial palsy without any detectable convulsions. Her EEG showed widespread spike-wave discharges, and computed tomographic analysis revealed a suspected small infarct in the median cerebral artery region contralateral to the facial palsy.
Of the 8 patients with epileptic seizures, 2 had their convulsions during recovery from the BDG operation. They each had a cerebral infarct, with convulsions and hemiplegia as presenting symptoms. Neither the type of a shunt in the Norwood operation $(23.1 \%$ in the BT group and $20.0 \%$ in the right ventricle group) nor the support times were associated with the occurrence of seizures.

\section{Predictors of Neurodevelopmental Outcome}

In the risk factor analysis with DQ as the primary outcome measure, those patients who had postoperative seizures and a focal EEG abnormality $(\mathrm{n}=7)$ had significantly lower mean DQ results than the others (77.3 vs 99.9, $P=.006$; Figure E1). Five $(71.4 \%)$ of them had a DQ more than 2 SDs below the mean of the control subjects compared with $4(16.0 \%)$ of 25 of all other patients $(P=.010)$. These patients with epileptic seizures also had a significantly smaller age- and sex-adjusted head circumference than those without seizures $(-1.8$ vs -0.5 [SD], $P=.004)$. Factors that did not significantly affect DQ included prenatal diagnosis (available for 5 patients with HLHS and 3 patients with UVH), history of a Norwood-type operation, use of an RV-PA versus BT shunt in the Norwood operation, and an additional correction of the aortic arch afterward. Those 3 patients with circulatory collapse during the neonatal period caused by delayed diagnosis, as well as those 2 who had been resuscitated, did not have lower results than the others. None of the growth values at birth but the age- and sex-adjusted head circumference at 1 year of age correlated significantly with DQ among the patients (Pearson $=0.536$, $P=.002)$.

Factors related to support times and hypothermia and postoperative levels of diuresis did not correlate significantly with DQ. Apgar scores at 1 and 5 minutes correlated significantly with DQ among the patients (Spearman = $0.432, P=.014$, and Spearman $=0.512, P=.007$, respectively) but not among the control subjects. After BDG, those patients whose plasma lactate levels reached $2.5 \mathrm{mmol} / \mathrm{L}$ or 


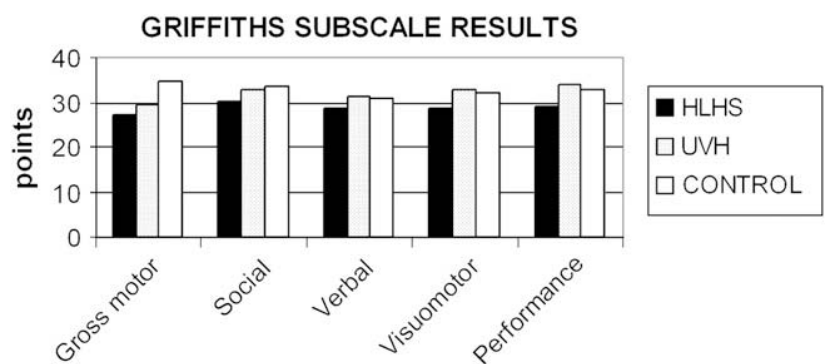

FIGURE 1. Griffiths developmental subscale results in patients with hypoplastic left heart syndrome (HLHS), patients with univentricular heart $(U V H)$, and healthy control subjects.

higher during the first 3 postoperative days had a significantly lower mean DQ than those with a lower lactate level ( 81.8 vs $98.0, P=.030)$. The DQ correlated negatively with the natural logarithm transformation of the highest plasma lactate levels 0 to 6 hours (Pearson $=-0.375, P=.034), 6$ to 24 hours (Pearson $=-0.438, P=.012$ ), and 0 to 72 hours $($ Pearson $=-0.464, P=.007)$ after BDG (Figure 3). Neither maternal or paternal occupational nor educational levels correlated significantly with DQ among either the patients or the control subjects, but because paternal educational level approached significance for the overall group (Spearman $=$ $0.209, P=.068$ ), it was used in the linear regression model to adjust for socioeconomic status.

In the multiple linear regression analysis adjusted for sex and level of paternal education, the HLHS diagnosis $(P=$ .016), clinical seizure history with abnormal EEG results $(P=.002)$, and natural logarithm transformation of the highest plasma lactate level in the first 3 postoperative days after $\mathrm{BDG}(P=.045)$ were significantly associated with the primary outcome of DQ, with an explanation rate of $54.2 \%$.

\section{DISCUSSION}

Our follow-up study showed that the development of children with HLHS and UVH at age 12 months was most disturbed in the motor area, but the children with HLHS had significantly lower results than control subjects in many other fields of development as well. The results from this population undergoing surgical intervention after the implementation of RLFP are comparable with earlier results, where the mean full-scale intelligence quotient has ranged from 84 to 94 in patients with HLHS and from 89 to 107 in patients with other forms of UVH. ${ }^{9-12,19,20}$ These earlier reports are cross-sectional retrospective studies of patients who were operated on at the time when DHCA was the support method in reconstructing the aortic arch. Our findings, compared with our earlier report on neurodevelopmental outcome of children with HLHS or UVH at age 5 to 7 years, ${ }^{11}$ suggest some improvement in the neurodevelopmental outcomes of these patients. This might apply especially to patients with UVH, even if the Griffiths DQ at

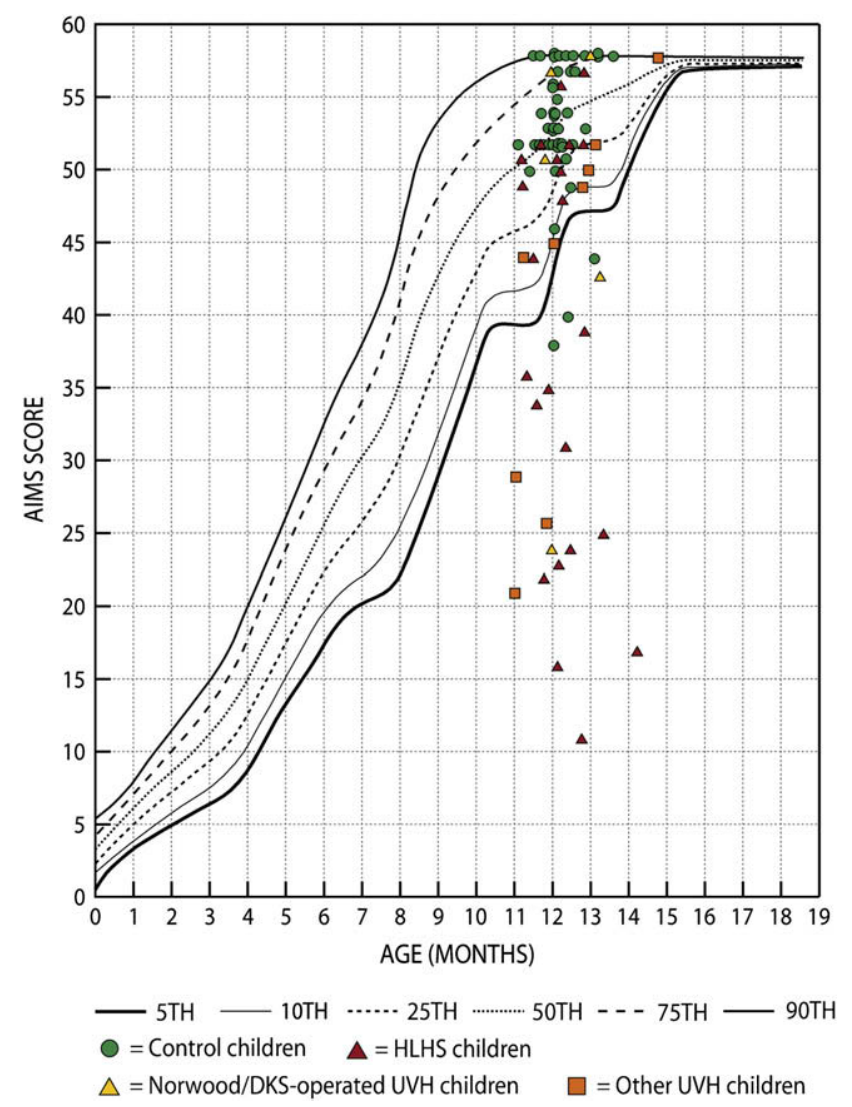

FIGURE 2. Alberta Infant Motor Scale (AIMS) results in patients with hypoplastic left heart syndrome (HLHS), patients with other forms of univentricular heart $(U V H)$ undergoing the Norwood operation, other patients with UVH, and control children at a median age of 12.2 months (range, 11.0 14.7 months). DKS, Damus-Kaye-Stansel.

age 1 year and full-scale intelligence quotient results at age 5 to 7 years cannot be directly compared. In addition to the use of RLFP instead of longer periods of DHCA, this improvement might be due to several other factors in the development of the preoperative, perioperative, and postoperative care of these patients.

We found that the development of these children is particularly impaired in the area of gross motor function at age 1 year. A similar trend toward lower motor performance compared with mental development among patients undergoing the Norwood operation at age 1 to 2 years has been shown by other investigators as well. ${ }^{5,6,21,22}$ Among older children with HLHS $^{9}$ and in other forms of congenital heart disease, ${ }^{23,24}$ motor deficits are also common. This might be a consequence of the vulnerability of certain brain areas, but at age 1 year, the delay in gross motor function might in part be explained by the immobilization related to the 1 to 2 operations performed during the first year of life and by muscle hypotonia.

An increased frequency of microcephaly occurs among patients with HLHS ${ }^{9,12}$ or other forms of congenital heart disease. ${ }^{24}$ Head circumference at birth correlates with 


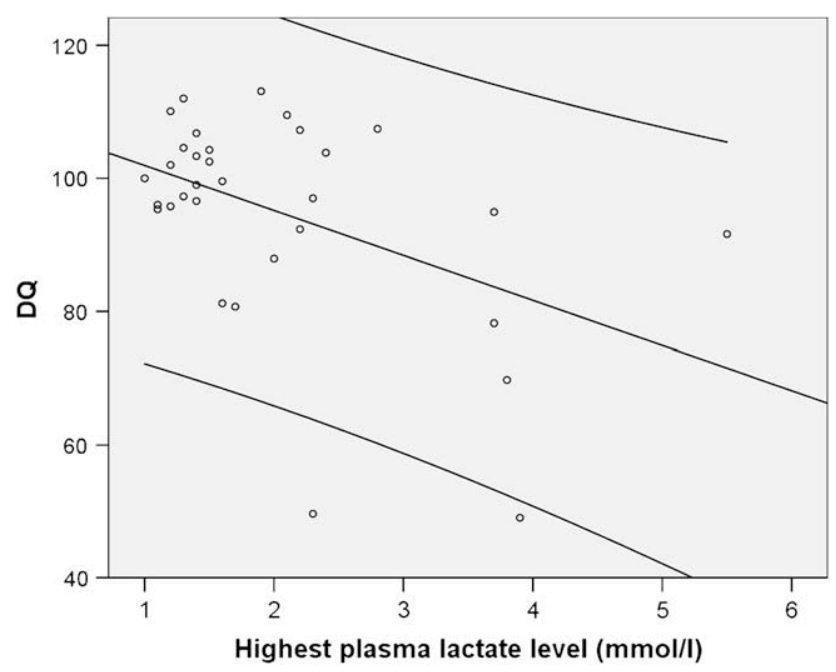

FIGURE 3. Highest plasma lactate level (in millimoles per liter) during the first 72 postoperative hours after the bidirectional Glenn operation and Griffiths developmental quotient $(D Q)$ at a median age of 12.2 months in 32 patients. Outer lines represent the $95 \%$ confidence interval.

ascending aortic diameter in children with HLHS, signifying diminished brain growth caused by their abnormal circulation. ${ }^{25}$ In our study population microcephaly was not as common as in some earlier reports, and mean head circumference at birth did not differ from that of control subjects. By contrast, at 1 year of age, the incidence of microcephaly remained the same, but mean head circumference was significantly lower in patients than in control subjects, which is in contrast to weight and height. Among patients, but not among control subjects, age- and sex-adjusted head circumference correlated significantly with DQ and was also strongly associated with any seizures experienced. Correlation of head size with DQ can be explained by diminished brain growth as a result of ischemic brain damage. This is confirmed by the fact that after exclusion of the 2 patients with HLHS with global ischemic damage, the correlation was no longer significant.

Of the risk factors examined, diagnosis of HLHS, clinical seizures with abnormal EEG activity, and increased postoperative plasma lactate levels after BDG surgery were significantly associated with outcome. Patients with HLHS have been shown to be at higher risk for neurodevelopmental sequelae than those with other forms of UVH. ${ }^{19,21}$ This difference can arise from several factors, such as impaired fetal and preoperative cerebral blood flow, possible structural brain anomalies, and the demanding Norwood operation, which is performed in only some patients with UVH. One limitation of our study is that the small number of patients with UVH might affect their results. In a few earlier retrospective reports, the occurrence of clinical preoperative ${ }^{9}$ or perioperative ${ }^{19}$ seizures has emerged among these patients as a predictor of worse outcome. The Boston Circulatory
Arrest Study found, among patients with transposition of the great arteries, a significant correlation between both clinical and electroencephalographic postoperative seizures and worse psychomotor development at 1 year of age, although not with mental development. ${ }^{26}$ Meanwhile, other investigators have found no significant correlation between electroencephalographic seizures and early neurodevelopmental outcome; however, in this case the EEG seizures were treated on the basis of clinical discretion, which might have reduced their detrimental effect on development. ${ }^{27}$ Whether the seizures lead to brain damage as such or reflect underlying brain damage remains debatable. Among many of our patients, clinical seizures were one sign of more profound neurologic damage, a fact also neuroradiologically evident.

High serum lactate levels were associated with mortality in several reports. ${ }^{28}$ As an indicator of tissue hypoxia, lactate could be assumed to associate with neurodevelopmental outcome as well, but reports of such an association among survivors of congenital heart surgery are few. In one study the time needed for the plasma lactate levels to decrease to 2 $\mathrm{mmol} / \mathrm{L}$ was longer among those with adverse neurodevelopmental outcome than among intact survivors, although not significantly so (16 vs 11 hours, $P=.07$ ). ${ }^{29}$ Among 34 patients after correction of total anomalous pulmonary venous return, the highest plasma lactate level correlated significantly with psychomotor, but not with mental, development at age 18 to 24 months. ${ }^{30}$ We found a significant correlation between plasma lactate concentration during the first 3 postoperative days after the BDG operation and global development at age 1 year. The correlation existed even though the lactate levels were only mildly increased. Why this was not true for the relatively higher lactate levels after the Norwood operation remains unknown.

We conclude that at 1 year of age, children with HLHS had significantly lower developmental results in most areas examined, whereas the development of children with UVH was significantly inferior to that of their control subjects in the motor area only. The neurologic abnormalities were usually mild but significantly more frequent among patients with HLHS than among control subjects. In addition to the HLHS diagnosis, a history of postoperative clinical seizures and the highest plasma lactate levels during the first 3 postoperative days after the BDG operation were significant predictors of outcome.

We thank Ritva Haajanen, PT, for her valuable work in the motor assessment of these children, and professor Seppo Sarna for statistical advice and reviewing the statistical analysis of this study.

\section{References}

1. Tweddell JS, Hoffman GM, Mussatto KA, Fedderly RT, Berger S, Jaquiss RD, et al. Improved survival of patients undergoing palliation of hypoplastic left heart syndrome: lessons learned from 115 consecutive patients. Circulation. 2002; 106(suppl 1):82-9. 
2. Sano S, Ishino K, Kawada M, Arai S, Kasahara S, Asai T, et al. Right ventriclepulmonary artery shunt in first-stage palliation of hypoplastic left heart syndrome. J Thorac Cardiovasc Surg. 2003;126:504-10.

3. Pigula FA, Nemoto EM, Griffith BP, Siewers RD. Regional low-flow perfusion provides cerebral circulatory support during neonatal aortic arch reconstruction. J Thorac Cardiovasc Surg. 2000;119:331-9.

4. Wypij D, Newburger JW, Rappaport LA, duPlessis AJ, Jonas RA, Wernovsky G, et al. The effect of duration of deep hypothermic circulatory arrest in infant heart surgery on late neurodevelopment: the Boston Circulatory Arrest Trial. J Thorac Cardiovasc Surg. 2003;126:1397-403.

5. Visconti KJ, Rimmer D, Gauvreau K, del Nido P, Mayer JE Jr, Hagino I, et al. Regional low-flow perfusion versus circulatory arrest in neonates: one-year neurodevelopmental outcome. Ann Thorac Surg. 2006;82:2207-13.

6. Goldberg CS, Bove EL, Devaney EJ, Mollen E, Schwartz E, Tindall S, et al. A randomized clinical trial of regional cerebral perfusion versus deep hypothermic circulatory arrest: Outcomes for infants with functional single ventricle. J Thorac Cardiovasc Surg. 2007;133:880-7.

7. Cua CL, Thiagarajan RR, Gauvreau K, Lai L, Costello JM, Wessel DL, et al. Early postoperative outcomes in a series of infants with hypoplastic left heart syndrome undergoing stage I palliation operation with either modified Blalock-Taussig shunt or right ventricle to pulmonary artery conduit. Pediatr Crit Care Med. 2006;7:238-44.

8. Tabbutt S, Dominguez TE, Ravinshankar C, Marino BS, Gruber PJ, Wernovsky G, et al. Outcomes after the stage I reconstruction comparing the right ventricular to pulmonary artery conduit with the modified Blalock Taussig shunt. Ann Thorac Surg. 2005;80:1582-91.

9. Mahle WT, Clancy RR, Moss EM, Gerdes M, Jobes DR, Wernovsky G. Neurodevelopmental outcome and lifestyle assessment in school-aged and adolescent children with hypoplastic left heart syndrome. Pediatrics. 2000;105:1082-9.

10. Mahle WT, Visconti KJ, Freier MC, Kanne SM, Hamilton WG, Sharkey AM, et al. Relationship of surgical approach to neurodevelopmental outcomes in hypoplastic left heart syndrome. Pediatrics. 2006;117:e90-7.

11. Sarajuuri A, Jokinen E, Puosi R, Eronen M, Mildh L, Mattila I, et al. Neurodevelopmental and neuroradiologic outcomes in patients with univentricular heart aged 5 to 7 years: related risk factor analysis. J Thorac Cardiovasc Surg. 2007; 133:1524-32.

12. Creighton DE, Robertson CM, Sauve RS, Moddemann DM, Alton GY, NettelAguirre A, et al. Neurocognitive, functional, and health outcomes at 5 years of age for children after complex cardiac surgery at 6 weeks of age or younger. Pediatrics. 2007;120:e478-86.

13. Kantero RL, Tiisala R. Studies on growth of Finnish children from birth to 10 years V. Growth of head circumference from birth to 10 years. A mixed longitudinal study. Acta Paediatr Scand Suppl. 1971;220:27-32.

14. Sorva R, Tolppanen EM, Perheentupa J. Variation of growth in length and weight of children. I. Years 1 and 2. Acta Paediatr Scand. 1990;79:490-7.

15. Griffiths R. The abilities of babies. A study in mental measurement. High Wycombe, United Kingdom: Association for Research in Infant and Child Development. 1986.
16. Piper MC, Darrah J. Motor assessment of the developing infant. Philadelphia, Pa: W.B. Saunders Company; 1994.

17. International standard classification of occupations, International Labor Office (ILO). Geneva: International Labor Office; 1990.

18. UNESCO international standard classification of education (ISCED). Paris: UNESCO; 1997.

19. Goldberg CS, Schwartz EM, Brunberg JA. Neurodevelopmental outcome of patients after the Fontan operation: a comparison between children with hypoplastic left heart syndrome and other functional single ventricle lesions. J Pediatr. 2000 137:646-52.

20. Wernovsky G, Stiles KM, Gauvreau K, Gentles TLFRACP, duPlessis AJ, Bellinger DC, et al. Cognitive development after the Fontan operation. Circulation. 2000;102:883-9.

21. Tabbutt S, Nord AS, Jarvik GP, Bernbaum J, Wernovsky G, Gerdes M, et al Neurodevelopmental outcomes after staged palliation for hypoplastic left heart syndrome. Pediatrics. 2008;121:476-83.

22. Robertson CM, Joffe AR, Sauve RS, Rebeyka IM, Phillipos EZ, Dyck JD, et al Outcomes from an interprovincial program of newborn open heart surgery. J Pediatr. 2004;144:86-92

23. Majnemer A, Limperopoulos C, Shevell M, Rosenblatt B, Rohlicek C, Tchervenkov C. Long-term neuromotor outcome at school entry of infants with congenital heart defects requiring open-heart surgery. J Pediatr. 2006;148:72-7.

24. Dittrich H, Buhrer C, Grimmer I, Dittrich S, Abdul-Khaliq H, Lange PE. Neurodevelopment at 1 year of age in infants with congenital heart disease. Heart. 2003 89:436-41.

25. Shillingford AJ, Ittenbach RF, Marino BS, Rychik J, Clancy RR, Spray TL, et al Aortic morphometry and microcephaly in hypoplastic left heart syndrome. Cardiol Young. 2007;17:189-95.

26. Rappaport LA, Wypij D, Bellinger DC, Helmers SL, Holmes GL, Barnes PD et al. Relation of seizures after cardiac surgery in early infancy to neurodevelopmental outcome. Circulation. 1998;97:773-9.

27. Gaynor JW, Jarvik GP, Bernbaum J, Gerdes M, Wernovsky G, Burnham NB et al. The relationship of postoperative electrographic seizures to neurodevelopmental outcome at 1 year of age after neonatal and infant cardiac surgery. J Thorac Cardiovasc Surg. 2006;131:181-9.

28. Cheifetz IM, Kern FH, Schulman SR, Greeley WJ, Ungerleider RM, Meliones JN Serum lactates correlate with mortality after operations for complex congenital heart disease. Ann Thorac Surg. 1997;64:735-8.

29. Cheung PY, Chui N, Joffe AR, Rebeyka IM, Robertson CM. Postoperative lactate concentrations predict the outcome of infants aged 6 weeks or less after intracardiac surgery: a cohort follow-up to 18 months. Western Canadian Complex Pediatric Therapies Project, Follow-up Group. J Thorac Cardiovasc Surg. 2005;130: 837-43.

30. Alton GY, Robertson CM, Sauve R, Divekar A, Nettel-Aguirre A, Selzer S, et al. Early childhood health, growth, and neurodevelopmental outcomes after complete repair of total anomalous pulmonary venous connection at 6 weeks or younger. J Thorac Cardiovasc Surg. 2007;133:905-11. 


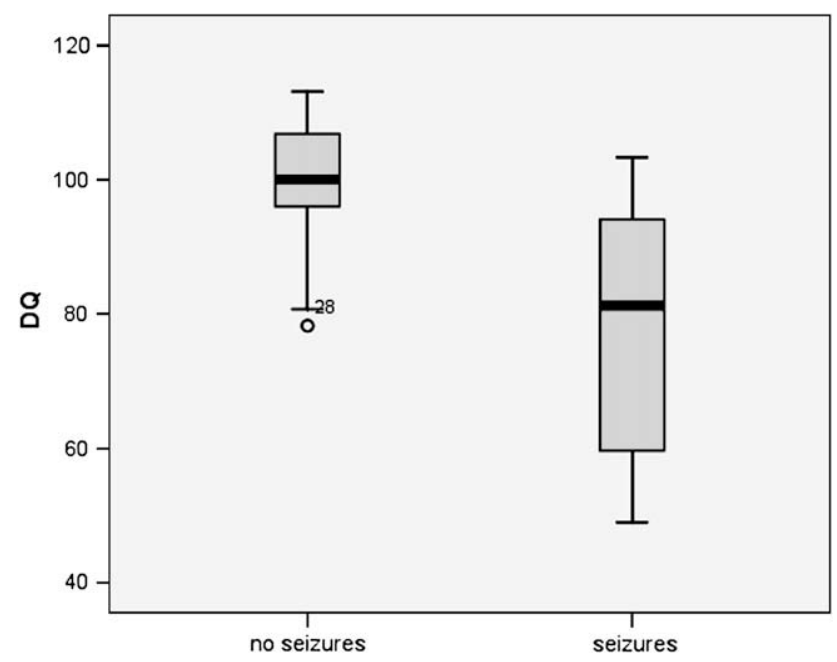

FIGURE E1. Griffiths developmental quotient $(D Q)$ values in patients with hypoplastic left heart syndrome/univentricular heart with $(\mathrm{n}=7)$ and without $(\mathrm{n}=25)$ epileptic seizures. Thick lines represent medians. The upper end of the box represents $75 \%$ of the patients, and the lower end represents $25 \%$ of the patients. 
TABLE E1. Patients with HLHS/UVH examined at a median age of 12.2 months $(n=37)$ : Diagnoses and operations performed

\begin{tabular}{|c|c|c|c|c|c|}
\hline & Age (d) & & & & \\
\hline & Median & Range & Operation & Diagnosis & $\mathbf{n}$ \\
\hline \multirow[t]{13}{*}{ Primary operation } & 7.0 & $1-37$ & Norwood & HLHS & 23 \\
\hline & & & Norwood & DILV, HAA, CoA, RV hypo & 1 \\
\hline & & & Norwood & TA, HAA, TGA, VSD & 1 \\
\hline & & & Norwood & AVSD, HAA, LV hypo & 1 \\
\hline & & & Norwood & Nonbalanced AVSD, CoA & 1 \\
\hline & & & $\begin{array}{l}\text { DKS, BT modification, } \\
\text { rec. aortic arch }\end{array}$ & DILV, TGA, HAA, CoA & 1 \\
\hline & & & CoA correction, PA banding & TA, dextrocardia, RV hypo, TGA, VSD & 1 \\
\hline & & & BT shunt & $\begin{array}{l}\text { AVSD, dextrocardia, TGA, RV hypo, } \\
\text { heterotaxy }\end{array}$ & 1 \\
\hline & & & BT shunt & PA+HVS, RV hypo & 3 \\
\hline & & & PA banding & TA, VSD, PA, RV hypo & 1 \\
\hline & & & BT shunt & TA, VSD, PS, RV hypo & 1 \\
\hline & & & BT shunt, septectomy & PAHVS, TA & 1 \\
\hline & & & No operation & DORV, dextrocardia, VSD, PS, MS, ASD & 1 \\
\hline BDG operation & 171.0 & $63-394$ & & & 37 \\
\hline \multirow[t]{8}{*}{ Additional operation } & 108.5 & $1-287$ & CoA correction & HLHS & 1 \\
\hline & & & LPA reconstruction, BT shunt & $\begin{array}{l}\text { AVSD, dextrocardia, TGA, RV hypo, } \\
\text { heterotaxy }\end{array}$ & 1 \\
\hline & & & Aortic valve plasty & HLHS & 1 \\
\hline & & & Re-BDG, thrombectomy & AVSD, LV hypo, HAA & 1 \\
\hline & & & PA banding & TA, VSD, PS, RV hypo & 1 \\
\hline & & & Septectomy and PA banding & HLHS & 1 \\
\hline & & & Re-BT shunt & $\mathrm{PA}+\mathrm{IVS}$ & 1 \\
\hline & & & LPA plasty, aortic arch plasty & HLHS & 1 \\
\hline \multirow[t]{2}{*}{ Second additional operation } & 203.5 & 99-308 & SVC plasty & HLHS & 1 \\
\hline & & & $\begin{array}{l}\text { Reconstruction of aorta } \\
\text { and PA }\end{array}$ & HLHS & 1 \\
\hline
\end{tabular}

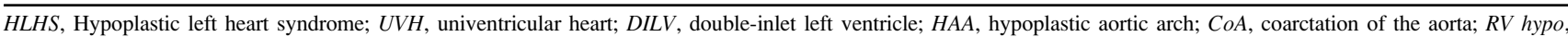
hypoplasia of the right ventricle; TA, tricuspid atresia; TGA, transposition of the great arteries; VSD, ventricular septal defect. AVSD, atrioventricular septal defect; $L V$ hypo, hypoplasia of the left ventricle; $D K S$, Damus-Kaye-Stansel; $B T$, Blalock-Taussig; $P A$, pulmonary artery; $P A H V S$, pulmonary atresia with intact ventricular septum; $P S$, pulmonary stenosis; $D O R V$, double-outlet right ventricle; $M S$, mitral stenosis; $A S D$, atrial septal defect; $B D G$, bidirectional Glenn operation; $L P A$, left pulmonary artery; $S V C$, superior vena cava. 